\title{
Intergenerational social mobility and allostatic load in Great Britain
}

\author{
Patrick Präg, ${ }^{1}$ Lindsay Richards ${ }^{2}$
}

- Additional material is published online only. To view please visit the journal online (http://dx.doi.org/10.1136/ jech-2017-210171)

${ }^{1}$ Department of Sociology, University of Oxford, Oxford, UK ${ }^{2}$ Centre for Social Investigation, Nuffield College and Department of Sociology, University of Oxford, Oxford, UK

\section{Correspondence to}

Dr Patrick Präg, Department of Sociology and Nuffield College, University of Oxford, Oxford OX1 3UQ, UK; patrick.prag@ sociology.ox.ac.uk

Previous versions of this article were presented at the 2017 Summer Meeting of RC28 in New York, a 2017 seminar at the Mannheim Center for European Social Research (MZES), the 2017 ECSR conference in Milan, the 2018 annual meeting of the PAA in Denver and a 2018 workshop at Nuffield College in Oxford.

Received 25 October 2017 Revised 18 September 2018

Accepted 7 October 2018 Published Online First

1 November 2018

\begin{abstract}
Background Intergenerational social mobility is hypothesised to be a stressful process that has a negative effect on health. By examining the relationship between own socioeconomic position, parental socioeconomic position and allostatic load (AL) in a representative sample of the British population, we test this hypothesis.

Methods Our study uses cross-sectional data from 9851 adult participants of waves 2 and 3 of Understanding Society. The relationship between parental occupational class at age 14 years, respondents' social class at the time of the interview and $A L$ is explored by means of diagonal reference models, which allow us to disentangle the effects of parental social class, own social class and the mobility process. The AL score comprises the following biomarkers: (1) total cholesterol, (2) high-density lipoprotein cholesterol, (3) triglycerides, (4) glycated haemoglobin, (5) C-reactive protein, (6) fibrinogen, (7) systolic blood pressure, (8) diastolic blood pressure, (9) resting heart rate, (10) body mass index and (11) waist circumference.

Results $\mathrm{AL}$ is particularly high among the stable working class and low among the stable upper class. On average, current class and origin class exert about equal weight on current AL. However, social mobilityregardless of whether upwards or downwards - is not detrimental for AL. Furthermore, we find evidence that class of origin may be less important among those outside the labour market for reasons other than retirement.
\end{abstract}

Conclusion Both own social class and parental social class influence $A L$ to a similar extent. However, we find no evidence that mobility trajectories exert any effects, good or bad, on $\mathrm{AL}$.

\section{INTRODUCTION}

Socioeconomic position (SEP) is a powerful predictor of individual health and well-being. Next to one's own position, the SEP of one's parents has separable and independent effects. These effects might be thought of in terms of cumulative advantage: the longer an individual spends in a high position, the longer the time without the stresses and strains associated with low SEP. ${ }^{12}$ Such life course approaches are effective in explaining all-cause mortality and mortality related to cardiovascular disease $^{3}$ as well as psychosocial functioning ${ }^{4}$ and self-rated health ${ }^{5}$ in adulthood.

However, while the socially stratified nature of health is well-established, there is a separate and unresolved question of whether intergenerational social mobility has an effect on health and well-being. To answer this question, social mobility must be considered in terms of its constituent parts of SEP in childhood (origin), SEP in adulthood (destination) and the trajectory of movement between the two. Research has shown that those in a lower SEP than their parents do poorly on outcomes including depressive symptoms and multisystemic dysregulation, and those in a higher SEP do well. ${ }^{67}$ However, these studies have generally not attempted to separate the effect of low SEP from the effect of downward mobility. Moreover, it has long been hypothesised that upward mobility can also be a stressful experience that is disruptive to social connections and attachments as individuals leave behind the social milieu they grew up in, ${ }^{8}$ in turn having negative health effects.

There is mixed evidence for this dissociative thesis, with some studies demonstrating a negative effect of upward mobility after controlling for current position ${ }^{9-11}$ while others have shown that upward mobility is conducive to higher wellbeing. ${ }^{612}$ However, others suggest both upward and downward mobility are beneficial, ${ }^{13}$ while some suggest that both upward and downward mobility have psychological costs ${ }^{10}$ or that only downward mobility is detrimental. ${ }^{14}$ Furthermore, mobility effects may also be asymmetrical. For example, the magnitude of losses to mental health of downward mobility are greater than the magnitude of the gains of upward mobility. ${ }^{6}$ In contrast, several studies find that any effect of mobility itself is absent or weak, ${ }^{1516}$ or depends on the context. ${ }^{91718}$

We identify two not mutually exclusive reasons for these discrepancies: the first is methodological-on which there is more discussion to follow. The second is that there is no agreed-upon standard about how the presence of dissociation should be inferred. While Daenekindt ${ }^{14}$ tackles the issue directly with a questionnaire instrument created to measure different facets of dissociation, most other studies rely on self-reported summary measures of mental and physical well-being, such as life satisfaction, ${ }^{9}$ happiness ${ }^{19}{ }^{20}$ or self-rated health ${ }^{21}$ and interpret a significant effect of mobility parameters as an indicator of the presence of dissociation. Next to validity concerns about self-reported outcome variables, ${ }^{22}$ the problem of adaptation or 'entrenched deprivation, ${ }^{23}$ may come into play. Qualitative research supports this latter point, ${ }^{10}$ implying that self-reported outcomes are subject to a cognitive bias. Our main aim in this study is to add to this debate by examining an objective indicator of well-being.

One framework within which to understand the effects of social mobility on health is the conceptual 
model of allostatis. ${ }^{24}$ Allostasis is a compensatory physiological mechanism that enables adaptation to psychosocial stressors to be able to regain physiological balance (homeostatis). The repeated activation of the stress response can however lead to a multisystem predisease state, characterised by the dysregulation of neuroendocrine, metabolic, inflammatory or cardiovascular systems. Allostatic load (AL) is thus the 'wear and tear' exacted on the body over time by efforts to adapt to life experiences. AL is strongly correlated with subclinical conditions as well as morbidity and mortality and is seen as a useful summary measure of overall health. ${ }^{25}$ Our study explores whether upward and/or downward social mobility affects AL. Previous studies of the dissociative thesis have focused on preventive healthcare use ${ }^{26}$ or self-reported measures of health and well-being..$^{9-21}$ Ours is the first to examine the effect of class dissociation on $\mathrm{AL}$, an objective indicator of well-being.

Distinguishing the health effect of the actual mobility experience from the health conditions characteristic of the early life SEP and the later-life SEP is not possible with conventional regression models and for this reason we make use of diagonal reference models (DRM). A secondary aim of our study is to understand the relative influences exerted by early life SEP and the later-life SEP on AL. Furthermore, we will explore the heterogeneity of class origin effects by mobility status. Since class is intertwined with labour market status, we also explore heterogeneous effects by working status, by age and by sex.

\section{METHODS}

\section{Data source and study population}

Understanding Society, the UK Household Longitudinal Study (UKHLS) is a prospective, nationally representative study, which incorporated the earlier British Household Panel Survey (BHPS). In waves 2 (2010-2012 for the UKHLS main sample) and 3 (2011-2012 for the BHPS), a large share of the study participants was sampled for nurse interviews, where physical measures, blood samples, and other health-related information were collected. The National Research Ethics Service approved the collection of biosocial data and informed consent was obtained from all participants. We analyse a sample of 9851 complete cases. Online supplementary file, table A1 presents case selection.

\section{Measurements \\ Social mobility}

We measure social mobility based on two indicators: own class and parental class. Respondents' own social class is based on current or, if not in the labour market, last occupation. Respondents were asked about their parents' occupations when respondents were aged 14 years. Occupation was coded according to the National Statistics Socioeconomic classification and collapsed to three classes: (i) working class, (ii) intermediate classes and (iii) salariat. For determining origin class, we rely on fathers' class and use mothers' class only in case father's class information is missing. As we are interested in destination class effects, we exclude young people (aged under 25 years) from the analysis, since they are unlikely to have reached occupational maturity.

\section{Allostatic load}

Our measure of AL comprises biomarkers that relate to secondary and tertiary stress responses. Primary responses such as cortisol could not be collected in the study context due to measurement difficulties, for example, time-of-day effects. Specifically, we make use of 11 biomarkers, categorised into 5 physiological systems: 1) lipid metabolism (total cholesterol, high-density lipoprotein cholesterol (values reversed) and triglycerides), 2) glucose metabolism (glycated haemoglobin), 3) inflammation (C-reactive protein and fibrinogen), 4) body fat deposition (body mass index and waist measurement) and 5) cardiovascular (systolic and diastolic blood pressure and resting heart rate). Despite wide variation in the operationalisation of $\mathrm{AL}$ in previous studies, ${ }^{27}$ our items cover the most frequently included measures.

As there is currently no agreement as to how AL should comprehensively be measured, we make use of two approaches. Our main approach is similar to Vie et al, ${ }^{28}$ which is based on a mean of the biomarker scores. We first z-transform our biomarkers, then calculate the mean score of the transformed biomarkers, and lastly z-transform that resulting score. For robustness checks, we use a different approach where we dichotomise the biomarkers once they surpass a clinically relevant, high-risk cut-off value (cut-off values reported in online supplementary file, table A2, see also Davillas and Pudney ${ }^{29}$ ). Our main measure captures greater variation as it is not reliant on clinical cut-offs, thereby accounting for the full range of predisease states, not just the 'elevated risk zone'. In both approaches, indicators are weighted by the number of parameters in the physiological system. The two AL measures correlate at $\mathrm{r}=0.74$.

\section{Covariates}

We account for age (centred around the sample mean of 52.6 years), sex $(0=$ male, $1=$ female $)$, ethnicity $(0=$ non-white, $1=$ white $), \quad$ partnership status $\quad(0=\mathrm{married} /$ partnered, $1=$ single/never married, $2=$ divorced/separated/widowed) and labour market status $(0=$ working, $1=$ retired, $2=$ not in labour market for another reason). Descriptive statistics for the covariates are presented in the online supplementary file, table A3.

\section{Statistical analysis}

We make use of diagonal reference models (DRM),${ }^{30-32}$ for the reason that conventional regression models are unable to identify social mobility effects. Being the difference between class of origin and destination, social mobility effects linearly depend on both of these parameters, leading to an identification problem. DRM has been used in health research before ${ }^{213}$ and recently van der Waal et $a l^{34}$ provided an important illustration of its value, but often other models are still used. ${ }^{7}$

DRMs estimate the effects of origin and destination social class on AL using a single vector of coefficients for both class positions along with weighting parameters representing the relative importance of the origin and destination classes:

$\mathrm{Y}=\mathrm{a}+\mathrm{p} \times \mu_{\mathrm{ii}}+\mathrm{q} \times \mu_{\mathrm{ij}}+\mathrm{bX}$

Where $a$ is the model intercept; subscripts $i$ and $j$ represent the social position of origin and destination, respectively; $\mu_{\mathrm{i}}$ and $\mu_{\mathrm{ij}}$ are both estimates of $\mathrm{Y}$ in the diagonal cells; $\mathrm{p}$ represents the relative importance of the class of origin and $\mathrm{q}$ the relative importance of the destination class, which is defined as $(1-p)$. X is a vector of covariates that can be interpreted like regression coefficients. The guiding assumption of DRM is that socially immobile individuals represent the most suitable point of reference, representing the true characteristics of that given class.

Analyses were conducted with the 'diagref' command in Stata V.13. A Stata do-file which allows replicating all analyses presented is available online (http://doi.org/10.17605/OSF.IO/ 6FUYJ). 


\begin{tabular}{|c|c|c|c|c|}
\hline \multirow[b]{2}{*}{ Origin class } & \multicolumn{2}{|c|}{ Destination class } & \multirow[b]{2}{*}{ Salariat } & \multirow[b]{2}{*}{ Total } \\
\hline & Working & Intermediate & & \\
\hline Working & $0.27(2080)$ & $0.09(1050)$ & $-0.01(1444)$ & 0.14 (4574) \\
\hline Intermediate & $0.10(894)$ & $-0.06(750)$ & $-0.13(1053)$ & $-0.04(2697)$ \\
\hline Salariat & $-0.04(540)$ & $-0.17(574)$ & $-0.30(1466)$ & $-0.21(2580)$ \\
\hline Total & 0.18 (3514) & $-0.02(2374)$ & $-0.15(3963)$ & 0.00 (9851) \\
\hline
\end{tabular}

\section{RESULTS}

Table 1 presents the average AL scores, with row and column totals revealing that $\mathrm{AL}$ is stratified both by origin class and destination class. The diagonal of the table reveals a stronger social gradient for the socially immobile. The AL for the stably working class respondents is more than a quarter (0.27) of a SD higher than the average (which is 0.00 due to standardisation), for those from the intermediate classes it is around average $(-0.06)$, and $-0.30 \mathrm{SD}$ lower for the stable salariat. For the socially mobile, table 1 shows that AL scores range in size between the scores of their counterparts who are stably in their classes of origin and destination.

Table 2 presents the results of diagonal reference models. The table can be interpreted as follows. The constant denotes average AL for an individual with 0 on all covariates, that is, is male, of average age, non-white, married and working. The class coefficients indicate the class-specific deviations from the constant. The origin weight ranges between 0 and 1 , with 0 indicating that the origin class plays no role for determining current $\mathrm{AL}$ and 1 indicating that it is only the origin (and not the destination) class that governs current AL.

The class coefficients in model (1) of table 2 confirm the social gradient in AL found in table 1, even after controls. Working class respondents fare worse $(0.22,95 \% \mathrm{CI} 0.19$ to 0.26$)$ than those from the intermediate classes $(-0.02,-0.06$ to 0.02$)$ and the salariat $(-0.20,-0.24$ to -0.17$)$. The origin weight is 0.49 (0.41 to 0.58$)$, indicating that origin class is roughly as important in determining $\mathrm{AL}$ as is destination class. The coefficients for the control variables reveal that $\mathrm{AL}$ is lower among women $(-0.21$, -0.25 to -0.18$)$, increases with age $(0.020 .02$ to 0.02$)$ and is lower among whites $(-0.15,-0.25$ to -0.05$)$. Singles have lower AL than the married and cohabiting $(-0.08,-0.14$ to -0.03$)$, and the divorced and widowed have higher $\mathrm{AL}(0.08,0.03$ to $0.13)$. The retired have lower AL than those working $(-0.11$, -0.17 to -0.05 ), and individuals out of the labour market for other reasons exhibit higher AL $(0.21,0.14$ to 0.28$)$.

Models (2) to (6) investigate how far social mobility is associated with AL. Model (2) shows that mobility in any direction is not associated with $\mathrm{AL}(-0.00,-0.04$ to 0.04$)$. Models (3) and (4) distinguish between upward and downward mobility and reveal that coefficients are close to 0 and have CIs that include 0 . Models (5) and (6) further distinguish between long-range and short-range mobility, which yield similar results, namely no mobility coefficients that reach conventional levels of statistical significance. Both the Akaike Information Criterion and Bayesian Information Criterion suggest that the best-fitting model is the parsimonious model (1), lending further weight to our conclusion that specific trajectories of class mobility provide no additional explanatory power after accounting for origin and destination position.

The analyses in table 3 further explore the great importance of the class of origin for different subgroups in our sample through the addition of interaction terms between the origin weight parameter and different covariates. Models (1) and (2) show that there are no differences in the importance of origin class for $\mathrm{AL}$ between men and women and for different age groups. Models (3) to (5) show that the importance of origin is the same for the socially mobile and for the immobile, regardless of whether it is upward or downward. Models (6) and (7) reveal a difference in the importance of origin weight when it comes to labour market status. The AL of those out of the labour market is determined to a greater extent by destination $(\mathrm{p}=0.19=0.53-0.34)$ than the $\mathrm{AL}$ of those who are retired or active in the labour market $(\mathrm{p}=0.53$, $95 \%$ CI 0.44 to 0.61 ).

\section{Sensitivity analyses}

To assess the robustness of our findings, we conducted a number of sensitivity analyses, the coding details and tables for which are in the online supplementary file. First, we ran the analysis with our alternative measure of AL (table A5). Second, we calculated class using the household dominance approach ${ }^{35}$ (table A7). Third, we stratified our analyses by sex (table A10) and age (tables A13, A14). Fourth, we added controls for health behaviours (table A11) and educational attainment ${ }^{36}$ (table A9). We also reran analyses excluding the unemployed (table A12). Across all of these checks our findings prove robust, although we find the AL gradient is steeper for women than for men. Furthermore, we examined the effect of mobility based on a 5-class schema (table A8), showing substantively similar findings. Lastly, we examined the physiological systems separately (table A6). All five systems are stratified, although with some differences regarding the importance of origin class. For lipid metabolism, inflammation and body fat deposition, the origin weight is similar to the main analysis. For the cardiovascular system $(0.26,-0.00$ to 0.54$)$ and glucose metabolism $(0.39$, 0.23 to 0.55 ), however, it is markedly lower, yet CIs are wide for these indicators.

\section{DISCUSSION}

Our study has confirmed that the lowest AL is found among the stable salariat and the highest among the stable working class. Furthermore, we have shown that, on average, origin class and destination class exert equal influence on one's current $\mathrm{AL}$, thus we suggest that the influence of childhood SEP is both substantial and higher than many previous studies have suggested. ${ }^{192137}$ However, once accounting for origin and destination position, we find that social mobility itself, in terms of the direction or distance travelled between childhood and adulthood, has no influence on AL. From this, we are able to surmise that the AL of an individual who starts out in the working class and ends up in the salariat will be equal to an individual who starts out in the salariat and ends up in the working class. Furthermore, since there is no effect of long-range or short-range mobility compared with stable class membership, our findings counter Sorokin's 'dissociative thesis', which postulated that social mobility — both upwards and downwards-is straining for individuals, causing 'mental diseases and nervousness, psychoses and neuroses ${ }^{8}{ }^{8}$ Instead, our results are compatible with cumulative advantage theory. ${ }^{14}$ The more of the life course spent in high socioeconomic position, with the material advantage that brings, the lower the AL.

Nonetheless, we also find that the weight of origin appears to be weaker for those outside the labour market, a pattern for which the mechanism is unclear, although it has been noted elsewhere that employment status matters more for mental health 


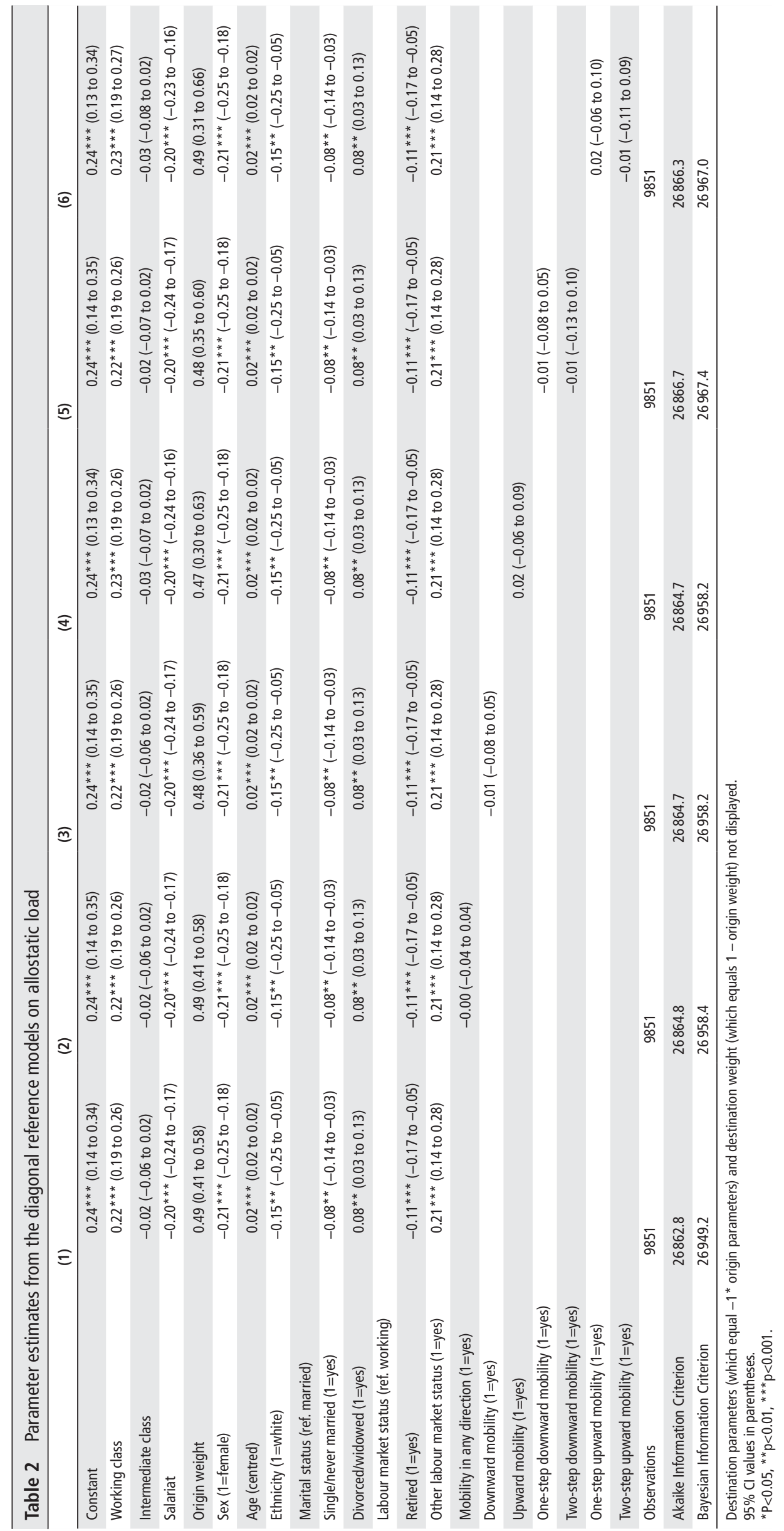


Table 3 Parameter estimates from the diagonal reference models on allostatic load, origin weights and interactions only

\begin{tabular}{|c|c|c|c|c|c|c|c|}
\hline & (1) & (2) & (3) & (4) & (5) & (6) & (7) \\
\hline & Female & Age & Mobile & $\begin{array}{l}\text { Downwardly } \\
\text { mobile }\end{array}$ & $\begin{array}{l}\text { Upwardly } \\
\text { mobile }\end{array}$ & Retired & Other job status \\
\hline Interaction term & $\begin{array}{l}0.09 \\
(-0.08 \text { to } 0.26)\end{array}$ & $\begin{array}{l}-0.00 \\
(-0.01 \text { to } 0.00)\end{array}$ & $\begin{array}{l}-0.00 \\
(-0.09 \text { to } 0.08)\end{array}$ & $\begin{array}{l}0.04 \\
(-0.19 \text { to } 0.28)\end{array}$ & $\begin{array}{l}-0.04 \\
(-0.28 \text { to } 0.19)\end{array}$ & $\begin{array}{l}-0.05 \\
(-0.24 \text { to } 0.14)\end{array}$ & $\begin{array}{l}-0.34^{*} \\
(-0.67 \text { to }-0.02)\end{array}$ \\
\hline Origin weight & $\begin{array}{l}0.44^{* * *} \\
(0.32 \text { to } 0.57)\end{array}$ & $\begin{array}{l}0.49^{* * *} \\
(0.41 \text { to } 0.58)\end{array}$ & $\begin{array}{l}0.50 \\
(0.50 \text { to } 0.50)\end{array}$ & $\begin{array}{l}0.48^{* * *} \\
(0.35 \text { to } 0.60)\end{array}$ & $\begin{array}{l}0.52^{* * *} \\
(0.35 \text { to } 0.68)\end{array}$ & $\begin{array}{l}0.51^{* * *} \\
(0.41 \text { to } 0.60)\end{array}$ & $\begin{array}{l}0.53^{* * *} \\
(0.44 \text { to } 0.61)\end{array}$ \\
\hline Observations & 9851 & 9851 & 9851 & 9851 & 9851 & 9851 & 9851 \\
\hline Akaike Information Criterion & 26863.75 & 26862.65 & 26862.85 & 26864.73 & 26864.73 & 26864.61 & 26858.44 \\
\hline Bayesian Information Criterion & 26957.28 & 26956.19 & 26949.19 & 26958.27 & 26958.27 & 26958.15 & 26951.98 \\
\hline
\end{tabular}

Origin parameters, destination parameters and control variables not displayed.

Full models displayed in online supplementary file, table A4.

$95 \% \mathrm{Cl}$ values in parentheses.

${ }^{*} \mathrm{P}<0.05,{ }^{* *} \mathrm{p}<0.01,{ }^{* * *} \mathrm{p}<0.001$.

than SEP, with the best outcomes seen among those in the workforce. ${ }^{38}$ We can speculate that individuals outside of the labour market are particularly vulnerable to effects of low income and social isolation, thus current social position has elevated significance.

\section{Limitations}

The length of time spent in both the class of origin and class of destination is likely to be important, but we are limited in ways to test this hypothesis. We find that the origin effect does not

\section{What is already known on this subject}

Socioeconomic position exerts a powerful effect on individual health and well-being.

- The socioeconomic conditions of childhood and adulthood have separable and additive effects.

- However, research on the effects of intergenerational social mobility has shown mixed and sometimes contradictory results.

- Studies of the health effects of social mobility so far have focused on subjective or self-rated outcomes.

\section{What this study adds}

- We examine allostatic load, an objective gauge of the 'wear and tear' that stress exerts on the body, which follows a pronounced social gradient.

- Lower allostatic load is seen among those experiencing socioeconomic advantage during childhood as well as in adulthood.

- Diagonal reference models allow us to discern the relative importance of origin and destination social class for allostatic load, as well as independent effects of specific mobility trajectories.

- We find that, on average, both origin and destination socioeconomic position exert about equal influence on current allostatic load.

- This is a stronger influence of origin than previous studies have suggested.

- Having accounted for socioeconomic position, however, social mobility per se, regardless of direction, has no influence on allostatic load. vary with age, but this may be an imperfect proxy for length of time in class. A second limitation is that with our research design we cannot account for AL baselines, or individual processes of adaption, or disease and so forth. The 'toxic stress' model, ${ }^{39}$ for example, postulates that adversity (including poverty) in childhood will influence occupational attainment and health independently. Addressing unmeasured heterogeneity of this sort may well be a fruitful direction for future research. Finally, with our summary measure of health we cannot be certain that there were no losses due to dissociation but only that they are outweighed by benefits of similar size.

\section{Methodological considerations}

A consensus is emerging in the literature that the diagonal reference model is superior to other modelling approaches and results based on other approaches are questionable at best. ${ }^{14} 1734 \mathrm{It}$ allows for an empirical breakdown of the components of social mobility, namely the starting position (origin), the finishing position (destination) as well as the particular trajectory of movement between the two. Previous studies have tended to apply an imperfect workaround. Examples include, first, including both origin and destination in a single model where significant origin estimates suggest lingering effects of childhood SEP. A second approach has been to create a series of dummy variables that capture mobility trajectories (eg, working class to salariat). This approach has illuminated the accentuated advantage of spending both childhood and adulthood in a high SEP but has not been able to disentangle the relative effects of childhood and adulthood positions.

An additional advantage of DRM is that it provides a means of explicitly estimating the relative influence of origin and destination on the outcome. In view of the known strong association between current SEP and health outcomes, our finding that the effect childhood SEP is just as strong, is both significant and consequential.

Acknowledgements The authors would like to thank discussants at presentations of this study as well as Nan Dirk de Graaf and Anthony Heath and the four anonymous JECH reviewers for comments. Understanding Society is an initiative funded by the Economic and Social Research Council and various government departments, with scientific leadership by the Institute for Social and Economic Research, University of Essex and survey delivery by Natcen Social Research and Kantar Public. The research data are distributed by the UK Data Service.

Contributors PP devised the study. LR and PP conducted the analyses and wrote the manuscript.

Funding The authors have not declared a specific grant for this research from any funding agency in the public, commercial or not-for-profit sectors. 
Competing interests None declared.

Patient consent Not required.

Provenance and peer review Not commissioned; externally peer reviewed.

Data sharing statement All data from the study are available at the UK Data Archive. Stata code to replicate the analyses is available on-line, DOI: 10.17605/OSF IO/6FUYJ.

\section{REFERENCES}

1 Gustafsson PE, Janlert U, Theorell T, et al. Socioeconomic status over the life course and allostatic load in adulthood: results from the Northern Swedish Cohort. J Epidemiol Community Health 2011;65:986-92.

2 Niedzwiedz CL, Katikireddi SV, Reeves A, et al. Economic insecurity during the great recession and metabolic, inflammatory and liver function biomarkers: analysis of the uk household longitudinal study. Journal of Epidemiology and Community Health 2018.

3 Stringhini S, Zaninotto P, Kumari M, et al. Socio-economic trajectories and cardiovascular disease mortality in older people: the english longitudinal study of ageing. Int J Epidemiol 2018;47:36-46

4 Harper S, Lynch J, Hsu WL, et al. Life course socioeconomic conditions and adult psychosocial functioning. Int J Epidemiol 2002;31:395-403.

5 Lindström M, Hansen K, Rosvall M. Economic stress in childhood and adulthood, and self-rated health: a population based study concerning risk accumulation, critical period and social mobility. BMC Public Health 2012;12:761.

6 Dolan P, Lordan G. Moving up and sliding down: an empirical assessment of the effect of social mobility on subjective wellbeing. London: CEP Discussion Papers CEPDP1190, 2013.

7 Na-Ek N, Demakakos P. Social mobility and inflammatory and metabolic markers at older ages: the English Longitudinal Study of Ageing. J Epidemiol Community Health 2017;71:253-60.

8 Sorokin PA. Social Mobility. Glencoe IL: Free Press, 1927.

9 Hadjar A, Samuel R. Does upward social mobility increase life satisfaction? A longitudinal analysis using British and Swiss panel data. Res Soc Stratif Mobil 2015;39:48-58.

10 Friedman $\mathrm{S}$. The price of the ticket: rethinking the experience of social mobility. Sociology 2014;48:352-68.

11 Gaydosh L, Schorpp KM, Chen E, et al. College completion predicts lower depression but higher metabolic syndrome among disadvantaged minorities in young adulthood. Proc Natl Acad Sci U S A 2018;115:109-14.

12 Chan TW. Social mobility and the well-being of individuals. Br J Sociol 2018:69:183-206.

13 Goldthorpe JH. Social Mobility and Class Structure in Modern Britain. Oxford: Clarendon, 1980.

14 Daenekindt S. The experience of social mobility: social isolation, utilitarian individualism, and social disorientation. Soc Indic Res 2017:133:15-30.

15 Marshall G, Firth D. Social mobility and personal satisfaction: evidence from ten countries. Br J Sociol 1999;50:28-48.

16 Iveson $\mathrm{MH}$, Deary IJ. Intergenerational social mobility and subjective wellbeing in later life. Soc Sci Med 2017;188:11-20.

17 Houle JN, Martin MA. Does intergenerational mobility shape psychological distress? Sorokin revisited. Res Soc Stratif Mobil 2011;29:193-203.

18 Gugushvili A, McKee M, Murphy M, et al. Intergenerational mobility in relative educational attainment and health-related behaviours. Soc Indic Res 2018;22
19 Zang E, de Graaf ND. Frustrated achievers or satisfied losers? Inter- and intragenerational social mobility and happiness in China. Sociol Sci 2016;3:779-800.

20 Zhao Y, Li Y, Heath A, et al. Inter- and intra-generational social mobility effects on subjective well-being - Evidence from mainland China. Res Soc Stratif Mobil 2017;48:54-66.

21 Monden CW, de Graaf ND. The importance of father's and own education for self-assessed health across Europe: an East-West divide? Sociol Health IIIn 2013;35:977-92.

22 Harris KM, Schorpp KM. Integrating biomarkers in social stratification and health research. Annu Rev Sociol 2018;44:361-86.

23 Sen A. Inequality Reexamined. Cambridge MA: Harvard University Press, 1992.

24 McEwen BS, Stellar E. Stress and the individual. Mechanisms leading to disease. Arch Intern Med 1993;153:2093-101.

25 Juster RP, McEwen BS, Lupien SJ. Allostatic load biomarkers of chronic stress and impact on health and cognition. Neurosci Biobehav Rev 2010;35:2-16.

26 Missinne S, Daenekindt S, Bracke P. The social gradient in preventive healthcare use: what can we learn from socially mobile individuals? Sociol Health IIIn 2015;37:823-38

27 Johnson SC, Cavallaro FL, Leon DA. A systematic review of allostatic load in relation to socioeconomic position: Poor fidelity and major inconsistencies in biomarkers employed. Soc Sci Med 2017;192:66-73.

28 Vie TL, Hufthammer KO, Holmen TL, et al. Is self-rated health a stable and predictive factor for allostatic load in early adulthood? Findings from the Nord Trøndelag Health Study (HUNT). Soc Sci Med 2014;117:1-9.

29 Davillas A, Pudney S. Concordance of health states in couples: analysis of self-reported, nurse administered and blood-based biomarker data in the UK Understanding Society panel. J Health Econ 2017;56:87-102.

30 Sobel ME. Diagonal mobility models: a substantively motivated class of designs for the analysis of mobility effects. Am Socio/ Rev 1981;46:893-906.

31 Sobel ME. Social mobility and fertility revisited: some new models for the analysis of the mobility effects hypothesis. Am Sociol Rev 1985;50:699-712.

32 Lizardo 0, 2007. diagref program to fit gaussian, logit, and poisson regression models that incorporate Sobel's (1981) non-linear parameterization of 'status inconsistency' effects for two identically coded independent variables $\mathrm{R}$ and $\mathrm{C}$ www3.nd.edu/ olizardo/Stataprogs/diagref/

33 Claussen B, Smits J, Naess O, et al. Intragenerational mobility and mortality in Oslo: social selection versus social causation. Soc Sci Med 2005;61:2513-20.

34 van der Waal J, Daenekindt S, de Koster W. Statistical challenges in modelling the health consequences of social mobility: the need for diagonal reference models. Int J Public Health 2017:62:1029-37.

35 Erikson R. Social class of men, women and families. Sociology 1984;18:500-14.

36 Präg P, Subramanian SV. Educational inequalities in self-rated health across US states and European countries. Int J Public Health 2017;62:709-16.

37 Schuck B, Steiber N. Does intergenerational educational mobility shape the well-being of young europeans? Evidence from the European Social Survey. Soc Indic Res 2018;139:1237-55.

38 Richards L, Paskov M. Social class, employment status and inequality in psychological well-being in the UK: Cross-sectional and fixed effects analyses over two decades. Soc Sci Med 2016;167:45-53.

39 McEwen CA, McEwen BS. Social structure, adversity, toxic stress, and intergenerational poverty: an early childhood model. Annual Review of Sociology 2017:43:445-72 